\title{
Hydrogen Bonds and Hydrophobic Interactions of Porphyrins in Porphyrin-Containing Proteins
}

\author{
Srđan Đ. Stojanović ${ }^{1}$ and Snežana D. Zarié ${ }^{* 2}$ \\ ${ }^{I}$ ICTM-Department of Chemistry, University of Belgrade, Belgrade, Serbia \\ ${ }^{2}$ Department of Chemistry, University of Belgrade, Belgrade, Serbia
}

\begin{abstract}
Structures of porphyrin-containing proteins from the Protein Data Bank (PDB) Select January 2007, were searched in order to find and systematically characterize hydrogen bonds and hydrophobic interactions of porphyrins in proteins. The results revealed that every porphyrin is involved in at least one hydrogen bond, most of the porphyrins form several, while some of them form up to thirteen hydrogen bonds. In most of the hydrogen bonds propionate groups of porphyrins interact with side-chains of residues. The most frequently observed donor is side chain of arginine. Histidine, lysine, threonine, serine and tyrozine form substantial number of hydrogen bonds too. The study has revealed that hydrophobic interactions are common between porphyrin and protein. Side-chains hydrophobic interactions are more frequent than those with backbone. The average conservation score for the amino acids making hydrogen bonds (7.2) and hydrophobic interactions (7.3) is statistically significantly higher than for the amino acids that are not involved in noncovalent interactions (5.7) with the porphyrin, indicating importance of hydrogen bonds and hydrophobic interactions with the porphyrin.
\end{abstract}

Keywords: Hydrogen bond, hydrophobic interactions, porphyrin, protein stability, PDB, conservation score.

\section{INTRODUCTION}

A detailed description of hydrogen bonds has been a long standing goal in structural biology, as they are crucial for the structure and function of the proteins [1]. The directionality of the hydrogen bond is very important since it allows the chemist to control the geometry of the complexes and to design precisely complementary hosts for a given guest. Studies of the structures of small molecules [2,3], surveys of proteins and nucleic acid structures in the Protein Data Bank (PDB) [4-6], and calculations based on classical molecular dynamics simulations $[7,8]$ and density functional theory (DFT) [9-11] have provided considerable insight into the geometry of hydrogen bonds. In structural studies, however, the presence of hydrogen bonds is most often inferred rather than actually detected [12].

Hydrophobic interactions are considered to be essential in many systems, be it micelles, vesicles, colloids, membranes and transport; self-organization, polymer interactions, protein folding and ligand binding, nucleic acids, drug action, water-mediated organic reaction. Hydrophobic interactions belong to the most often cited intermolecular forces [1].

Studies of the metal center in heme proteins and model systems have shown that many factors, including noncovalent interactions, can play important roles in the properties of these metalloproteins [13, 14]. In our previous work we studied orientations of imidazoles axially

\footnotetext{
*Address correspondence to this author at the Department of Chemistry, University of Belgrade, Belgrade, Serbia; Tel: +381113336605 ; E-mail: szaric@chem.bg.ac.rs
}

coordinated to iron-porphyrin [15-18]. It was shown that the noncovalent interactions with the propionic groups are very important for the orientations of the imidazoles and that the conformations of the propionic groups have strong influence on these interactions $[15,17]$.

In the majority of different types of porphyrin-containing proteins, porphyrin is noncovalently bound [19-21]. In our previous work we showed that porphyrin $\pi$-system in metal complexes and in proteins could be involved in $\mathrm{CH} / \pi$ interactions [22, 23]. Searching structures of porphyrincontaining proteins from the Protein Data Bank it was revealed that $\pi$-system of every porphyrin ring is involved in $\mathrm{CH} / \pi$ interactions and most of the porphyrins are making several interactions. The amino acids involved in the interactions show high conservation score indicating that $\mathrm{CH} / \pi$ interactions play an important role in the porphyrinprotein stability [23].

Here we studied hydrogen bonds and hydrophobic interactions of porphyrins in proteins. Negatively charged propionic groups of heme porphyrin can make strong hydrogen bonds and these bonds can determine conformations of the groups. As it was mentioned above, the conformations of the propionic groups greatly influence the orientation of axially coordinated imidazoles [15, 17]. We used a nonredundant database of the Protein Data Bank (PDB), with 3,398 protein chains to examine systematically the occurrence and the role of hydrogen bonds and hydrophobic interactions of porphyrins.

\section{METHODS}

For this study we used the Protein Data Bank (PDB) Select January 2007 list of non-redundant protein chains 
(25\% threshold version, 3398 protein chains and 494625 amino acid residues) [24]. The following criteria were employed to assemble the set: (1) no theoretical model structures and no NMR structures were accepted, (2) only crystal structures with the resolution of $3.0 \AA$ or better and a crystallographic R-factor of $25.0 \%$ or lower were accepted, (3) crystal structures containing porphyrin were accepted.

If not already present, all hydrogen atoms were added and optimized using the program REDUCE [25] with default settings. Bayesian method was used for calculating rates of amino acid conservation scores. Homologues were collected from SWISS-PROT, max. number of homologues $=50$, number of PSI-BLAST iterations $=1$ (PSI-BLAST E-value $=$ 0.001) [26], scale representing the conservation scores (9 conserved, 1 - variable). For testing statistical significance of mean difference of conservation scores we used nonparametric Kolmogorov-Smirnov two-sample test.

Hydrogen bonds and hydrophobic interactions between porphyrin rings and surrounding amino acids involved were calculated by program LIGPLOT v.4.4.2 using the default
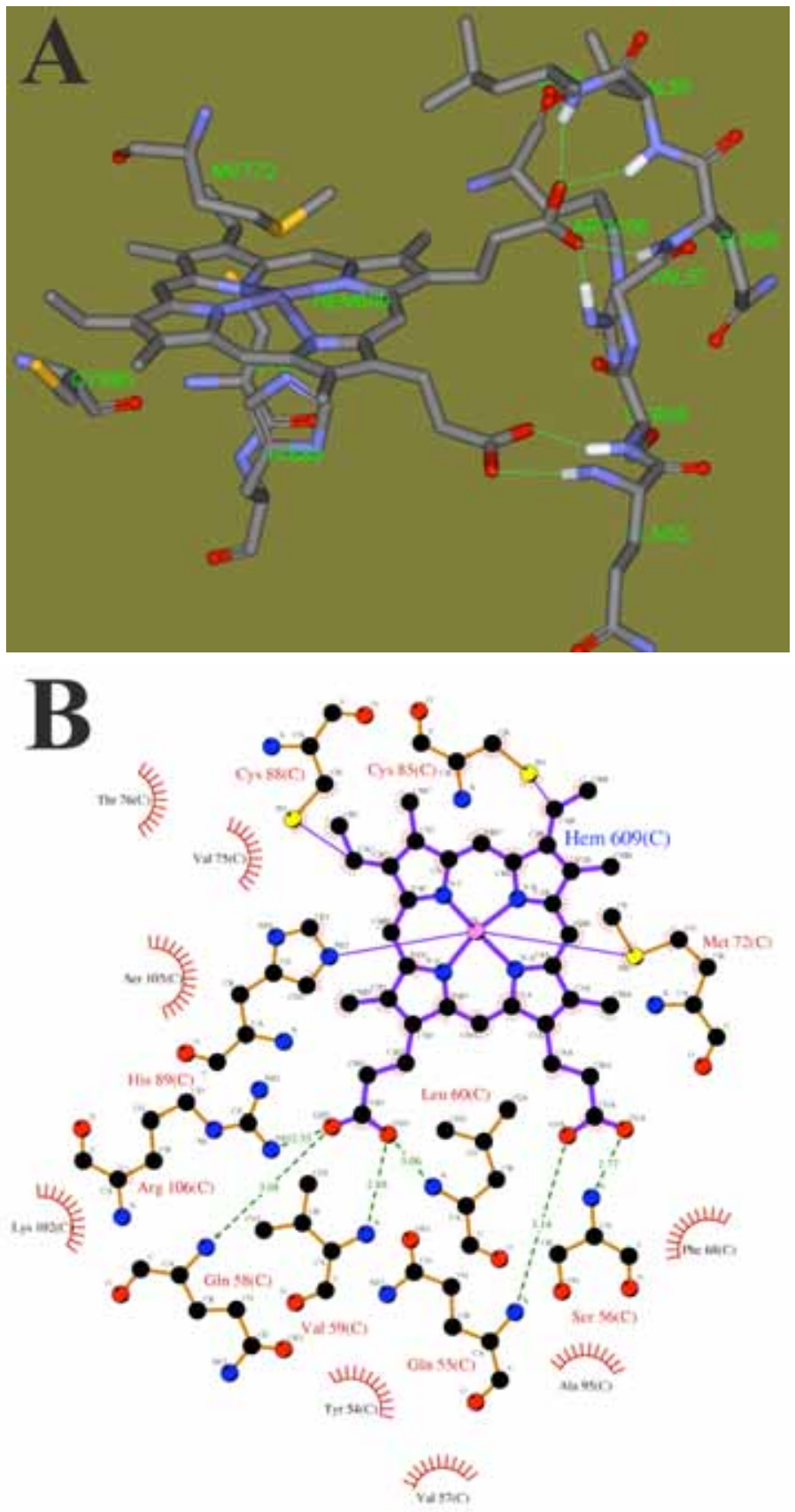

Fig. (1). View of the active site of photosynthetic reaction center from Thermochromatium tepidum (PDB ID code 1eys; HEM 609C). (A) $3 \mathrm{D}$ view of the active site. (B) The flattened diagram place atoms and bonds on the 2D page to minimize the overlap of atoms and the crossing of bonds in the final diagram. Hydrogen bonds are indicated by dashed green lines (with the distance between acceptor and donor atoms printed in the middle). Corresponding atoms involved in hydrophobic contacts are represented by an arc with spokes radiating towards the ligand atoms they contact. 
criterions [27]. The used criteria are: $\mathrm{H}-\mathrm{A}$ distance $<2.7 \AA$, $\mathrm{D}-\mathrm{A}$ distance $<3.3 \AA$, D-H-A angle $>90^{\circ}$, D-A-AA angle $>90^{\circ}$ and $\mathrm{H}-\mathrm{A}-\mathrm{AA}$ angle $>90^{\circ}$, where $\mathrm{AA}$ is the atom attached to the acceptor. All carbon atoms separated by $<3.9$ $\AA$ were considered to be interacting through hydrophobic contacts [27-29].

The Figures were prepared using programs LIGPLOT v.4.4.2 [27] and NOC Version 2.0.2 program by Chenmengen and H.X. Cang.

\section{RESULTS AND DISCUSSION}

In the nonredundant database of the PDB with 3,398 protein chains we found 57 proteins with porphyrin. Since some of the proteins contain more than one porphyrin in these proteins are 79 porphyrins. Using the geometrical criteria described in "Methods", we found out all of the hydrogen bonds and hydrophobic interactions of the porphyrins in the proteins.

An example of hydrogen bonds and hydrophobic interactions from Thermochromatium tepidum is shown in Fig. (1). There are six hydrogen bonds between porphyrin (HEM 609C) and surrounding amino acids (Gln55, Ser56, Gln58, Val59, Leu60 and Arg106) and 54 hydrophobic interactions.

\section{Hydrogen Bonds}

Using the geometrical criteria we found out that all of the porphyrins in the nonredundant database of the PDB form the hydrogen bonds. Some of the porphyrins form only one hydrogen bond (the structures with PBD ID codes lewa, $1 \mathrm{oj} 6,1 \mathrm{r} 2 \mathrm{c}, 2 \mathrm{bk} 9$ and 2czs), while most of the porphyrins form several hydrogen bonds. Some of the porphyrins have up to thirteen hydrogen bonds (some examples are structures with PBD ID codes 1eys, 1gwe, 1h10, 1h32, 1qks and 1qle).

\section{The Donor-Acceptor Types in Hydrogen Bonds}

Histograms of frequency of amino acid residues involved in hydrogen bonds with porphyrin via backbone and side chains are presented in Fig. (2). The hydrogen bonds between groups with net charge are well accepted in chemistry and biology [30-32], especially when the proton donor is positively charged. In our data (Fig. 2) most of the hydrogen bonds is formed with side chains of amino acids and also there are preference of particular amino acid side chains.

The number of hydrogen bonds, on the basis of data in the nonredundant database of the PDB, is 56 with backbone and 208 with side-chain hydrogen atoms. The backbone groups are not frequently involved, because of lacking the charge and because their atoms are not as accessible as the side-chain hydrogen atoms. As it can be anticipated, sidechains of the positively charged amino acids (Arg, His and Lys) the most frequently form hydrogen bonds.

Moreover, we found several interactions with side chains of Asp where Asp is defined as hydrogen donor. It is somewhat unexpected since the side chains of Asp are usually negatively charged. However, it is shown that in proteins there are pairs of hydrogen-bonded carboxylic acid side chains. Stable carboxyl-carboxilate pairs have quite short distance $(<2.7 \AA)$ between hydrogen donor and acceptor atoms [33]. We found out that the distances of two oxygens in propionic group-Asp interactions are in almost all cases below $2.7 \AA$.

The side chains of polar residues also participate in hydrogen bonds (Ser, Tyr and Thr), while hydrophobic residues are involved in hydrogen bonds only with their backbones.

The frequency of observed hydrogen bonds, sorted according atoms in the porphyrin are given in Fig. (3). The analysis has shown that most of the acceptors are carbonyl oxygen atoms (O1A, O2A, O1D and $\mathrm{O} 2 \mathrm{D})$ of propionate groups of porphyrins. Oxygen atoms $(\mathrm{O} 1 \mathrm{~B}, \mathrm{O} 2 \mathrm{~B}, \mathrm{O} 1 \mathrm{C}$ and $\mathrm{O} 2 \mathrm{C}$ ) of acetyl groups form much smaller number of hydrogen bonds, while very small fraction of acceptors are from the oxygen atom of other groups (oxo and hydroxyethyl).
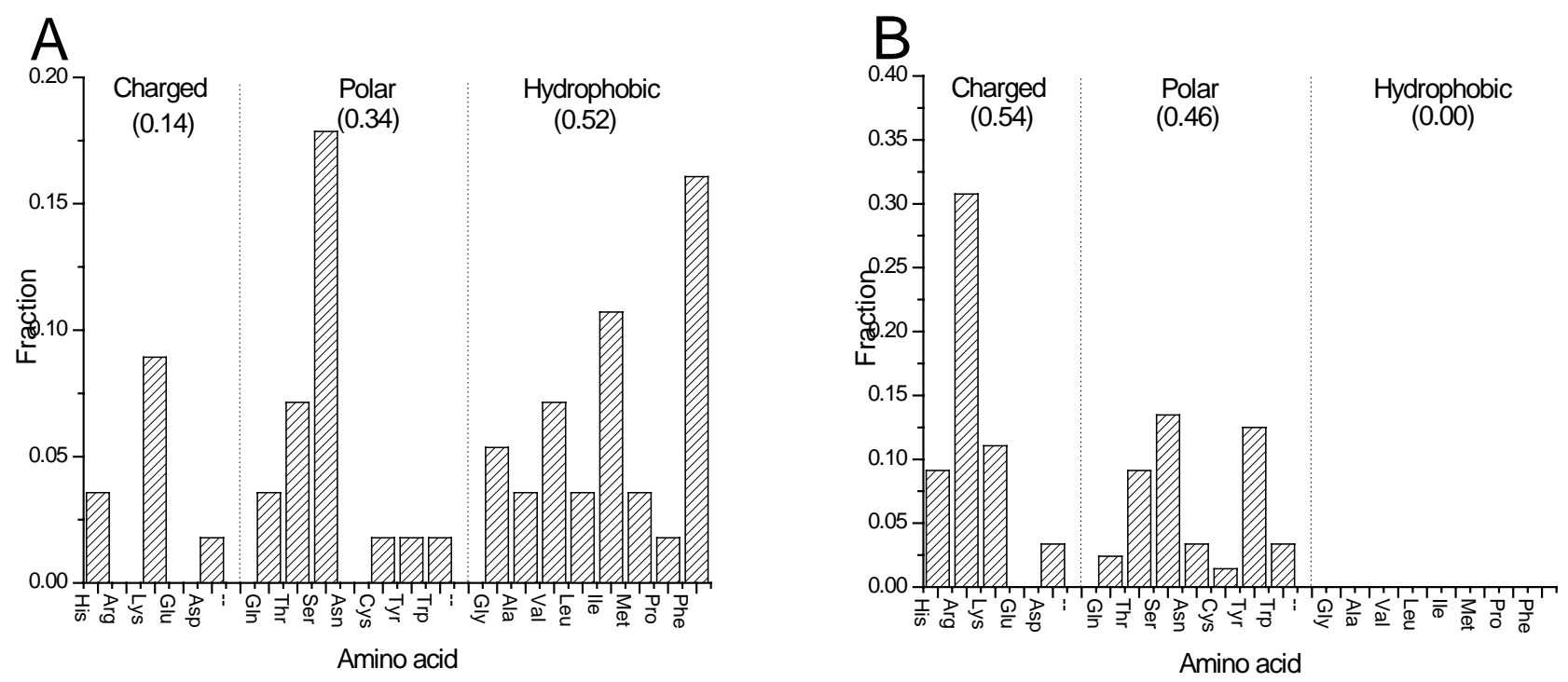

Fig. (2). Amino acid residue frequencies of donors in hydrogen bonds from the backbone (A) and from the side chains (B). 

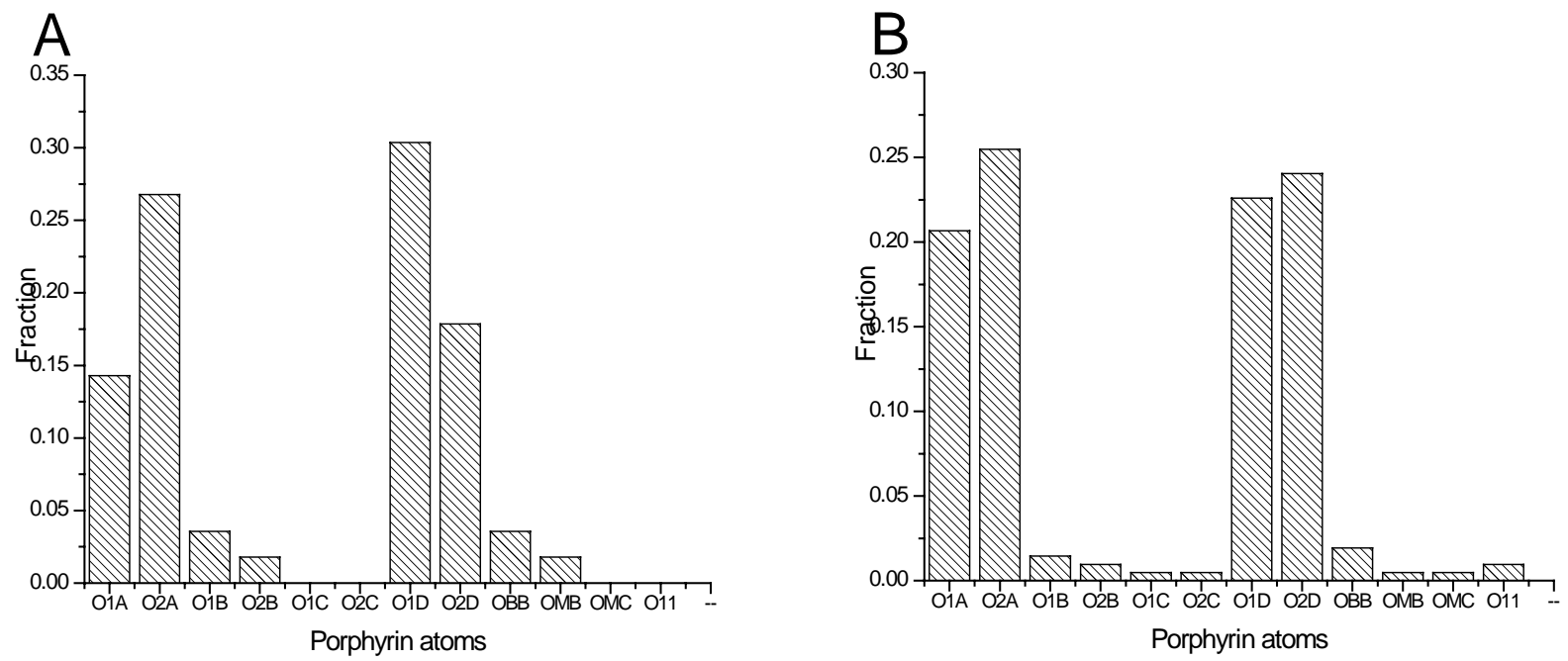

Fig. (3). Porphyrin atoms frequencies of acceptors in hydrogen bonds with the backbone (A) and with the side chains (B).

\section{Environment of the Hydrogen Bonds}

The results show that porphyrin in proteins form a significant number of hydrogen bonds and it is interesting to examine the environment of the hydrogen bonds. We analyzed the types of residue side chains that are present in the vicinity of the donor-acceptor pairs. All of the side chain atoms that occur within a distance of $4.5 \AA$ from the donor or the acceptor atom form the environment of the residue involved in the formation of hydrogen bond. The results presented in Fig. (4) indicate a high number of hydrophobic residues in the neighborhood of hydrogen bond.

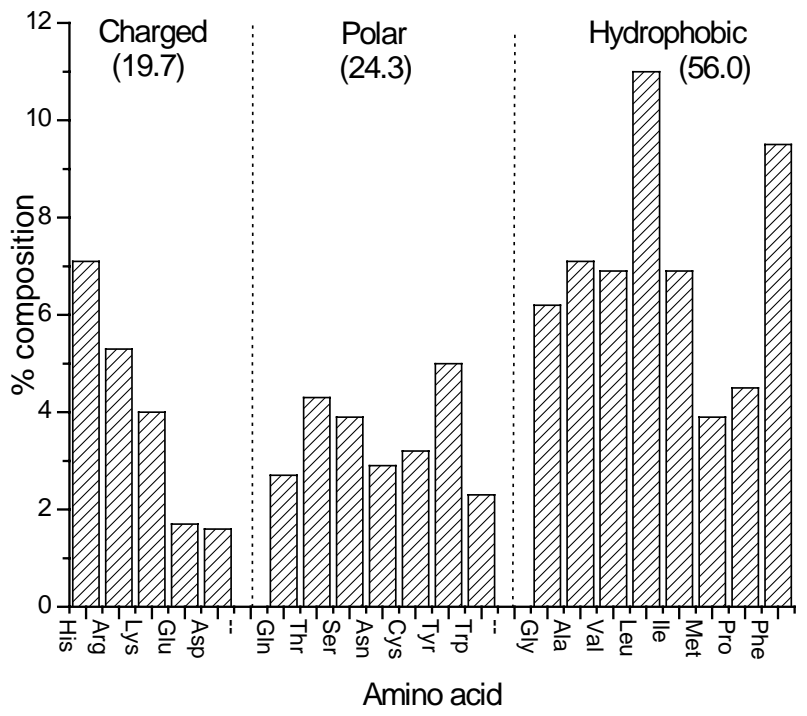

Fig. (4). Composition (\%) in the neighborhood of the residue involved in the formation of hydrogen bond.

Thus a large number of hydrogen bonds is in the hydrophobic environment. Furthermore, the presence of a larger number of aromatic side chains in the environment is also quite interesting. Hydrophobic environment and aromatic side chains are probable consequence of the hydrophobic pockets around porphyrins in proteins [34]. On other hand such hydrophobic environment could enhances electrostatic interactions (hydrogen bonds) by lowering in some way dielectric constant.

\section{Multiple and Bridged Hydrogen Bonds}

Hydrogen bonds with two donors (acceptor bifurcation) and two acceptors (donor bifurcation) are known to be common in protein structures $[35,36]$. The role of hydrogen bonds in recognition of the ligand by the protein has been studied and the donor and acceptor bifurcation has been analyzed in detail [37]. In our analysis, we have investigated the cases of donor (approach of many acceptors towards a donor) and acceptor (approach of many donors towards an acceptor) miltifurcated hydrogen bonds. The analysis shows that about $10 \%$ of the total hydrogen bonds in the dataset are involved in the formation of multiple hydrogen bonds. The details of donor and acceptor bifurcation are given in Table 1. There is a larger number of acceptor bifurcations. This could mainly be due lower geometrical constraints for acceptor bifurcation, as the two donor atoms are separated in space without sacrificing the hydrogen bond geometry.

Oxygen atoms of porphyrin can form hydrogen bonds with water molecules, and water can be bridge to the amino acid residues. It is known that water networks in protein interfaces can complement direct interactions contributing significantly to molecular recognition, function, and stability of protein association [38-40]. We have identified water molecules that are bridges between oxygen atoms of porphyrin and amino acids. Our results show 46 hydrogen bonds of the amino acid residues with porphyrin interacting through water, emphasizing the importance of the inclusion of solvent in protein-ligand interaction studies (Table 1).

Table 1. Number of Multiple and Bridged Hydrogen Bonds

\begin{tabular}{|c|c|c|c|}
\hline \multicolumn{2}{|c|}{ Type of Hydrogen Bonds } & Total \\
\hline \hline \multirow{2}{*}{ Multiple } & Acceptor bifurcation & 17 & \multirow{2}{*}{26} \\
\cline { 2 - 3 } & Donor bifurcation & 9 & \\
\hline \multirow{2}{*}{ Water-bridged } & Acceptor water-bridged & 21 & \multirow{2}{*}{46} \\
\cline { 2 - 3 } & Donor water-bridged & 25 & \\
\hline
\end{tabular}

The binding pocket for the subunits of lignin peroxidase from Basidiomycete phanerochaete, as illustrative example 


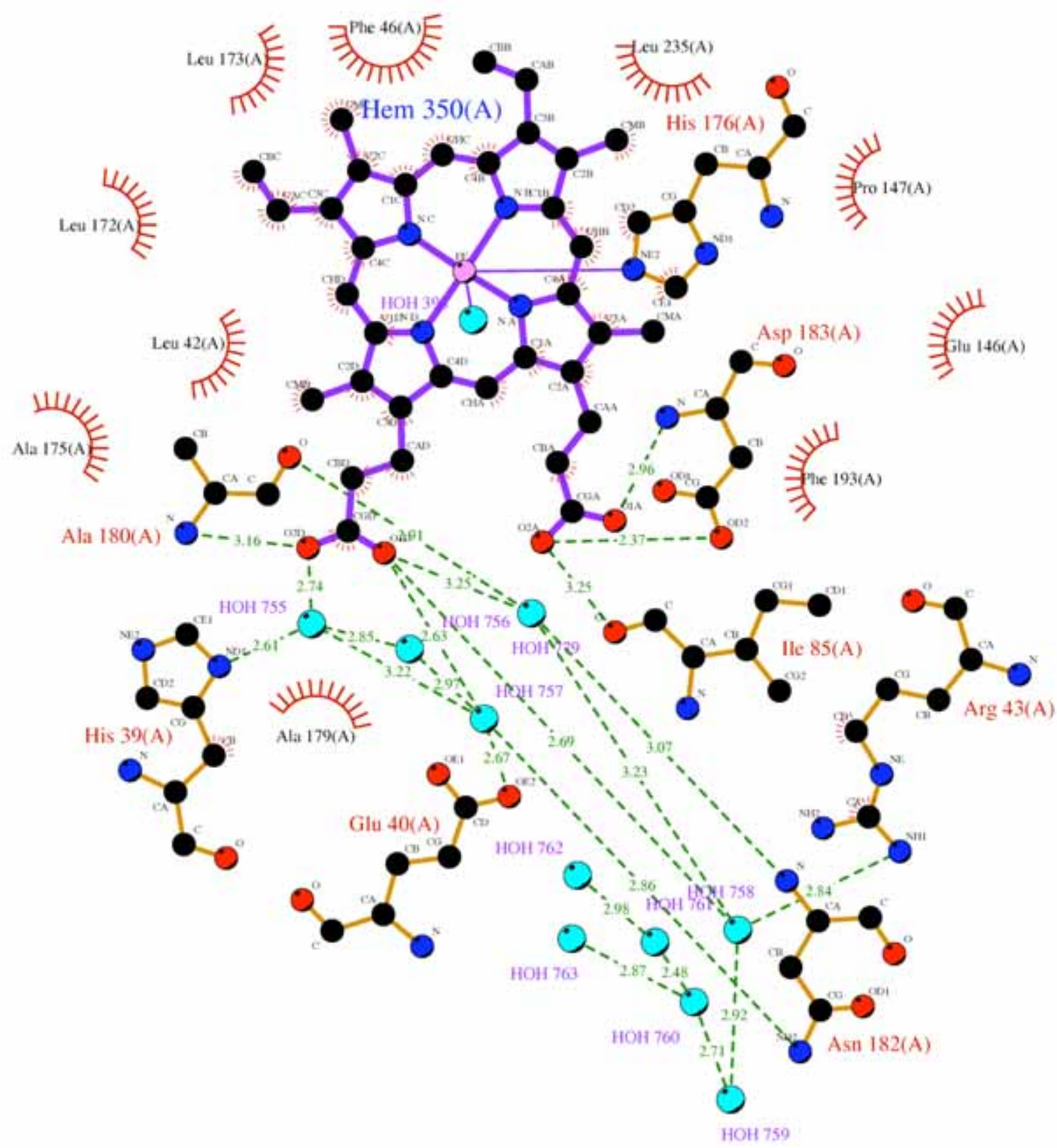

Fig. (5). View of the heme-binding pocket of lignin peroxidase from Basidiomycete phanerochaete (PDB ID code 1qpa). (For the explanation of the diagram see Fig. (1)). Water molecules are shown with cyan balls.

for bifurcation and water-bridged hydrogen bonds, is shown in Fig. (5). An example of acceptor bifurcated hydrogen bond is atom $\mathrm{O} 2 \mathrm{~A}$ from propionate group of porphyrin ring making hydrogen bonds with both Ile85 and Asp183. There are some water molecules that bridge hydrogen bonds between porphyrin ring and amino acid residues. One of them is HOH79 bridging hydrogen bond between atom O1D (porphyrin ring) and Asn 182.

\section{Hydrogen Bonds between Propionic Groups of Two Porphyrins}

It is very interesting that in the proteins that contain more than one porphyrin (PDB ID: 1fs9, 1h1o, 1m70, 1sp3, 2czs), the propionate groups of porphyrin can be involved in the hydrogen bonds with propionate groups of another porphyrin in the protein. We found six of those interactions. Three of them are hydrogen bond between propionate groups, while three of them are hydrogen bonds bridged by water molecule.

The binding pocket for the cytochrome c-552 from Thiobacillus ferrooxidans (PDB ID code 1h1o) with two iron-porphyrins is shown in Fig. (6). There is one hydrogen bond between porphyrins (Hem 1385 and Hem 1386) with distance of $3.05 \AA$. The same propionate groups of both porphyrins form hydrogen bonds with nearby amino acids (Arg335, Gln339, Tyr343 and Arg356) residues. 


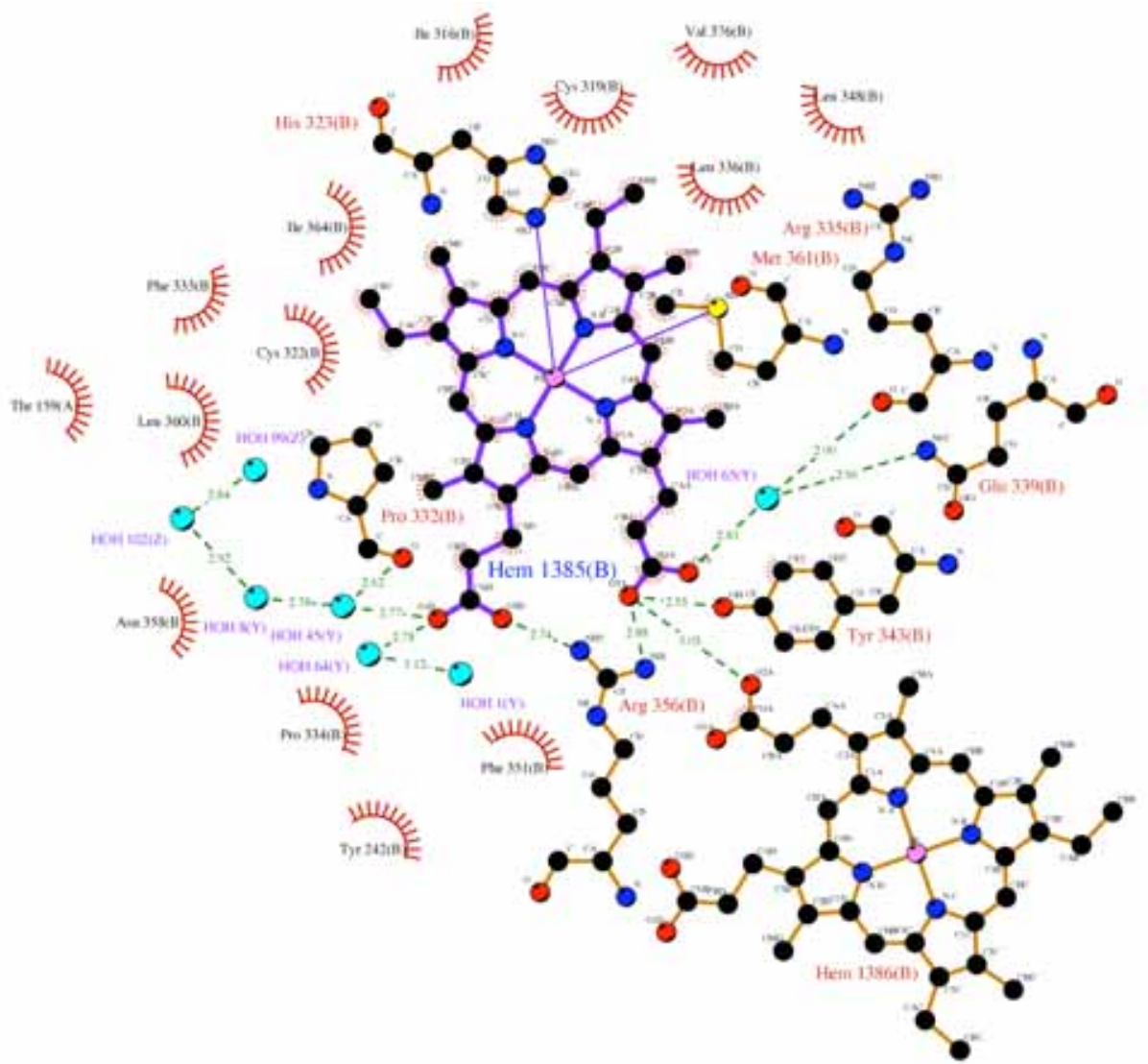

Fig. (6). Details of the hydrogen bond linking the porphyrins in the crystal of Thiobacillus ferrooxidans (PDB ID code 1h1o). (For the explanation of the diagram see Fig. (1)). Water molecules are shown with cyan balls.

\section{Donor-Acceptor Distances of the Hydrogen Bonds}

The analysis of the distance between the donor and the acceptor atoms is shown in Fig. (7). For hydrogen bonds of porphyrin with main chain the peak of the distribution is $2.88 \AA$, while for the hydrogen bonds with side chain bonds is $2.69 \AA$. The observed shorter distances for side chains is not surprising; probably due to steric reasons since the

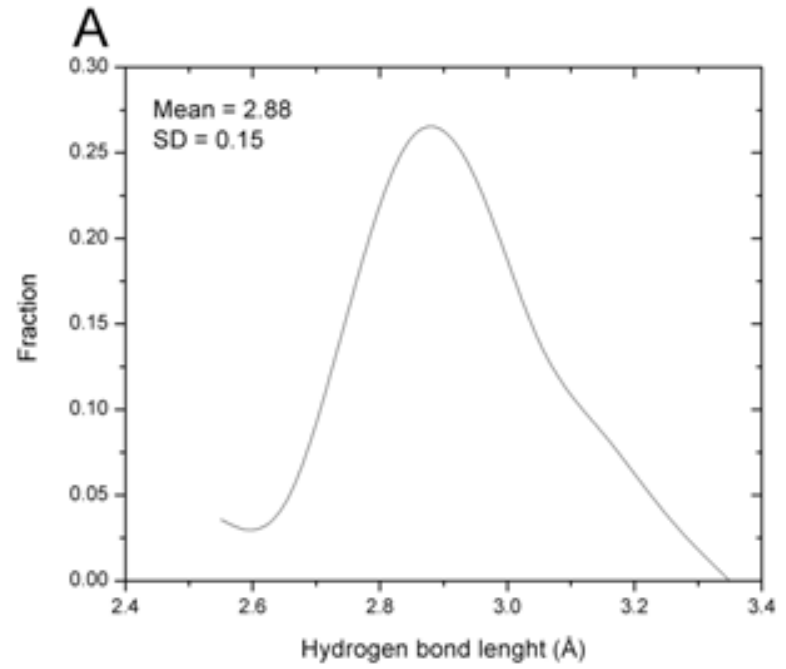

porphyrin ring is closer to the side-chain residues than the main-chain residues.

\section{Hydrophobic Interactions}

Occurrence of hydrophobic interactions, based on data in the non-redundant data base of the PDB, for different types of amino acids is shown in Fig. (8A).

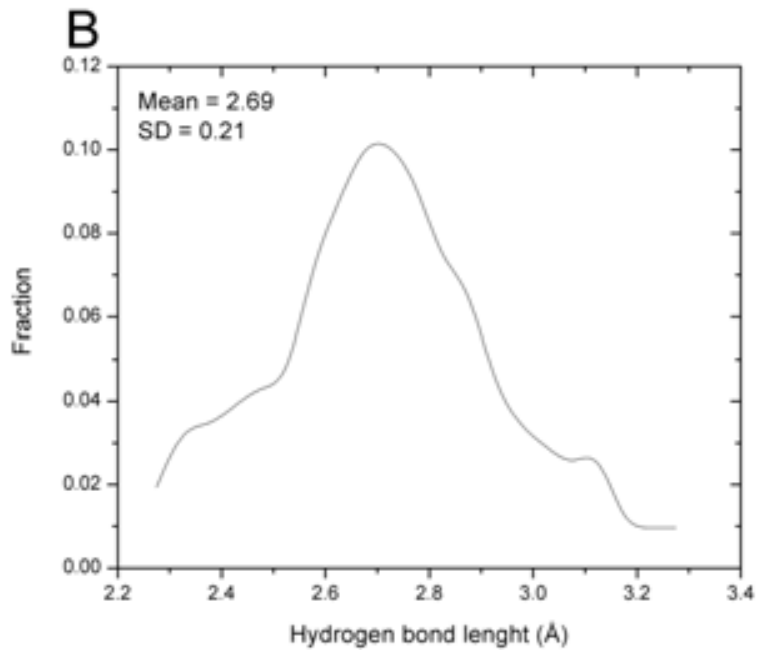

Fig. (7). Occurrence of the hydrogen-bonds as a function of the distance between the donor and the acceptor atoms of the main chain-side chain (A) and side chain-side chain (B) hydrogen bonds. 

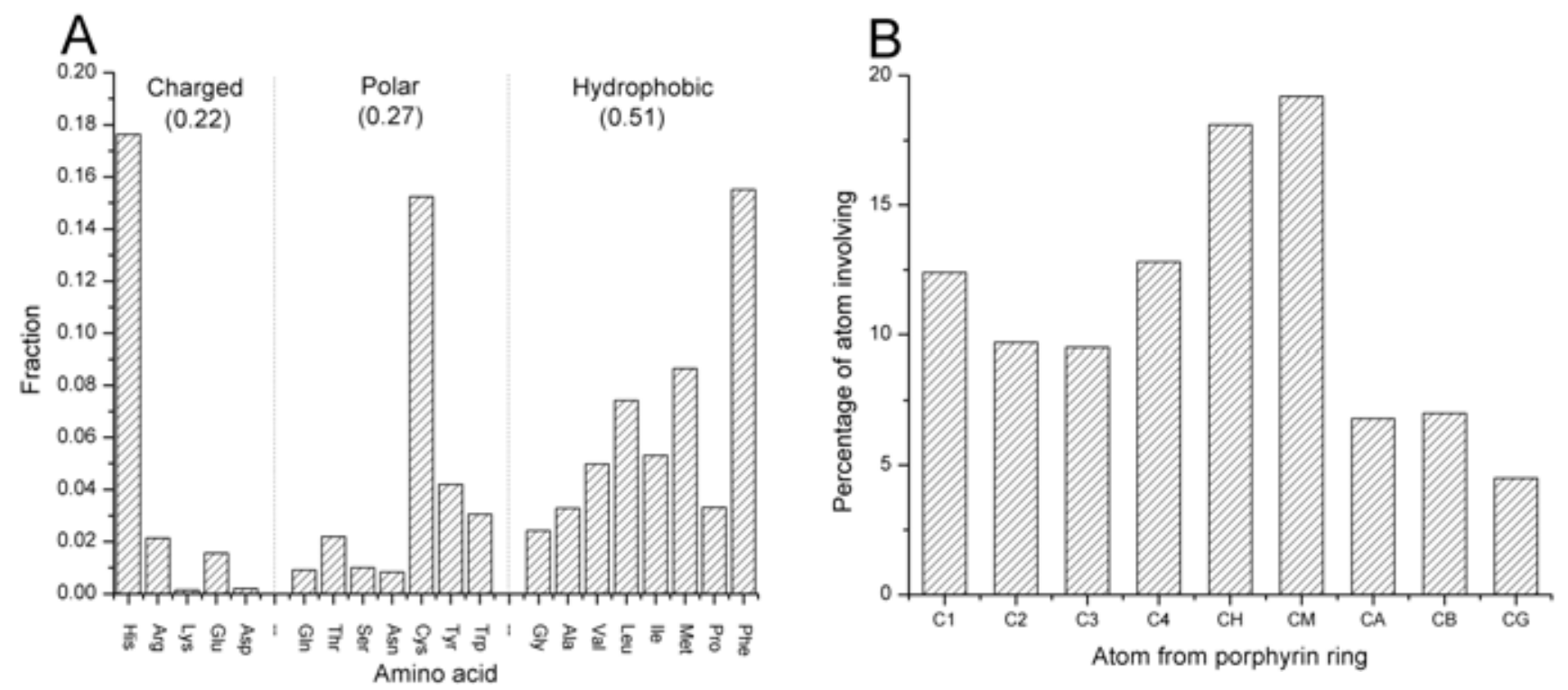

Fig. (8). (A) Frequency of amino acid residues involved in hydrophobic contacts with porphyrin rings, (B) The percentage of porphyrin ring atoms involved in hydrophobic contacts.

We found large number of hydrophobic interactions (3596) in porphyrin containing proteins. The results presented in Fig. (8A) show that the hydrophobic side chains make larger number of the interactions than side chains of charged and the polar amino acid. The backbone groups are not frequently involved (data not shown), because their atoms are not as accessible as the side-chain atoms.

We found that protein interactions with the hydrophobic face of the porphyrin play an important role in the stabilization of porphyrin-protein complexes (Fig. 8B). The most prominent porphyrin atoms involving in hydrophobic interactions are $\mathrm{CH}$ and $\mathrm{CM}$ from vinyl and methyl groups respectively because they are closer to the protein chain. The other atoms of porphyrin (C1, C2, C3 and C4) also significantly participate in hydrophobic interactions. Atoms of acetyl and propionate groups of porphyrin $(\mathrm{CA}, \mathrm{CB}$ and CG) have a less significant contribution of hydrophobic interactions.

\section{Conservation Score of Amino Acid Residues}

It is considered that structurally conserved residues are important in protein stability and folding [41]. In order to find out the importance of different noncovalent interactions of the porphyrin ring in proteins, in our previous work we studied the conservation of the residues involved in the interactions [23]. The data about the average conservation scores are calculated for four groups of amino acids in binding pocket; for the amino acids involved in the $\mathrm{XH} / \pi$ (X $=\mathrm{N}, \mathrm{C}$ ) interactions with porphyrin ring, in hydrogen bonds, in hydrophobic interactions, and for the amino acids that are not involved in any type of the interactions with the porphyrin [23].

By using a Bayesian method for calculating rates of amino acid conservation scores, we found that most of the amino acids involved in the hydrogen bonds are highly conserved: most of them have a conservation score of 9, the highest number on the scale. The calculated average conservation score for the amino acids making hydrogen bonds is 7.2. The amino acids forming hydrophobic interactions with porphyrin ring have similar average conservation scores; 7.3, indicating similar importance of hydrophobic and hydrogen bonds of porphyrins in proteins. The average conservation score of the amino acids that are not involved in any type of interaction with the porphyrin in the binding pocket is 5.7, and it is statistically significantly lower than for amino acids that form hydrogen bonds and hydrophobic interactions.

\section{CONCLUSIONS}

An analysis of all porphyrin-containing proteins from a nonredundant dataset has shown that large number of hydrophobic interactions exists between porphyrin and protein. Side-chains hydrophobic interactions are more frequent than those with main chain atoms. The high conservation score of amino acids involved in hydrophobic interactions is an additional strong argument for their importance.

The results also have shown that hydrogen bonds frequently occur with porphyrins, every porphyrin is forming hydrogen bond(s) with surrounding protein. As anticipated, many of them are found between positively charged groups of Arg, Lys, and His amino acids, with particularly large number of arginine residues forming hydrogen bonds. The polar groups also substantially contribute to hydrogen bonds. Moreover, hydrogen bonds are also observed with main chain atoms. Multifurcated and water-bridged hydrogen bonds have also been identified. In the proteins that contain more than one porphyrin, hydrogen bonds between propionic groups of two porphyrins can form. The analyzes of the types of residues in the vicinity of the hydrogen bonds indicate a high number of hydrophobic residues, that could enhances hydrogen bonds by lowering in some way dielectric constant. This observation, together with high conservation score of hydrogen bonding amino acids, additionally stands for the importance of these amino acids for formation of complex with porphyrin. All these findings 
may explain why certain amino acids were selected within evolutionary process of porphyrin binding pocket formation.

\section{ACKNOWLEDGEMENTS}

This work was supported by the Foundation of the Serbian Ministry of Science under grant no. 142037.

\section{REFERENCES}

[1] Atwood JL, Steed JW. Encyclopedia of Supramolecular Chemistry. Boca Raton: CRC Press 2004.

[2] Daber R, Stayrook S, Rosenberg A, Lewis M. Structural analysis of lac repressor bound to allosteric effectors. J Mol Biol 2007; 370: 609-19.

[3] Etter MC. Encoding and decoding hydrogen-bond patterns of organic-compounds. Acc Chem Res 1990; 23: 120-6.

[4] Gardberg AS, Dice LT, Ou S, et al. Molecular basis for passive immunotherapy of Alzheimer's disease. Proc Natl Acad Sci USA 2007; 104: 15659-64.

[5] Grishaev A, Bax A. An empirical backbone-backbone hydrogenbonding potential in proteins and its applications to NMR structure refinement and validation. J Am Chem Soc 2004; 126: 7281-92.

[6] McDonald IK, Thornton JM. Satisfying hydrogen bonding potential in proteins. J Mol Biol 1994; 238: 777-93.

[7] Buck M, Karplus M. Hydrogen bond energetics: a simulation and statistical analysis of $\mathrm{N}$-methyl acetamide (NMA), water, and human lysozyme. J Phys Chem B 2001; 105: 11000-15.

[8] Ogawa H, Nakano M, Watanabe H, Starikov EB, Rothstein SM, Tanaka S. Molecular dynamics simulation study on the structural stabilities of polyglutamine peptides. Comput Biol Chem 2008; 32: 102-10.

[9] Lipsitz RS, Sharma Y, Brooks BR, Tjandra N. Hydrogen bonding in high-resolution protein structures: a new method to assess NMR protein geometry. J Am Chem Soc 2002; 124: 10621-6.

[10] Sass HJ, Schmid FF, Grzesiek S. Correlation of protein structure and dynamics to scalar couplings across hydrogen bonds. J Am Chem Soc 2007; 129: 5898-903.

[11] Scheurer C, Bruschweiler R. Quantum-chemical characterization of nuclear spin-spin couplings across hydrogen bonds. J Am Chem Soc 1999; 121: 8661-2.

[12] Forrest LR, Honig B. An assessment of the accuracy of methods for predicting hydrogen positions in protein structures. Proteins 2005; 61: 296-309.

[13] Bertini I, Cavallaro G, Rosato A. Cytochrome c: occurrence and functions. Chem Rev 2006; 106: 95-115.

[14] Walker FA. Models of the bis-histidine-ligated electrontransferring cytochromes. Comparative geometric and electronic structure of low-spin ferro- and ferrihemes. Chem Rev 2004; 104: 589-615.

[15] Galstyan AS, Zarić SD, Knapp EW. Computational studies on imidazole heme conformations. J Biol Inorg Chem 2005; 10: 34354.

[16] Rakić A, Medaković V, Zarić S. Orientations of axially coordinated imidazoles and pyridines in crystal structures of model systems of cytochromes. J Inorg Biochem 2006; 100: 133-42.

[17] Zarić SD, Popović DM, Knapp EW. Factors determining the orientation of axially coordinated imidazoles in heme proteins. Biochemistry 2001; 40: 7914-28.

[18] Medaković V, Zarić S. Theoretical study on orientations of axially coordinated imidazoles in model systems of cytochromes. Inorg Chim Acta 2003; 349: 1-5.

[19] Arnesano F, Banci L, Bertini I, et al. The solution structure of oxidized Escherichia coli cytochrome $b_{562}$. Biochemistry 1999; 38 : 8657-70.
[20] Falzone CJ, Mayer MR, Whiteman EL, Moore CD, Lecomte JT. Design challenges for hemoproteins: the solution structure of apocytochrome $b_{5}$. Biochemistry $1996 ; 35: 6519-26$.

[21] Hargrove MS, Wilkinson AJ, Olson JS. Structural factors governing hemin dissociation from metmyoglobin. Biochemistry 1996; 35: 11300-9.

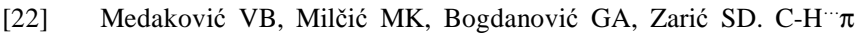
interactions in the metal-porphyrin complexes with chelate ring as the $\mathrm{H}$ acceptor. J Inorg Biochem 2004; 98: 1867-73.

[23] Stojanović SD, Medaković VB, Predović G, Beljanski M, Zarić $\mathrm{SD} . \mathrm{XH} / \pi$ interactions with the pi system of porphyrin ring in porphyrin-containing proteins. J Biol Inorg Chem 2007; 12: 106371.

[24] Hobohm U, Sander C. Enlarged representative set of protein structures. Protein Sci 1994; 3: 522-4.

[25] Word JM, Lovell SC. Asparagine and glutamine: using hydrogen atom contacts in the choice of sidechain amide orientation. J Mol Biol 1999; 285: 1735-47.

[26] Landau M, Mayrose I, Rosenberg Y, et al. ConSurf 2005: the projection of evolutionary conservation scores of residues on protein structures. Nucleic Acids Res 2005; 33: W299-302.

[27] Wallace AC, Laskowski RA, Thornton JM. LIGPLOT: a program to generate schematic diagrams of protein-ligand interactions. Protein Eng 1995; 8: 127-34.

[28] Sherman W, Tidor B. Novel method for probing the specificity binding profile of ligands: applications to HIV protease. Chem Biol Drug Des 2008; 71: 387-407.

[29] Blaszczyk-Thurin M, Murali R, Westerink MA, Steplewski Z, Co MS, Kieber-Emmons T. Molecular recognition of the Lewis Y antigen by monoclonal antibodies. Protein Eng 1996; 9: 447-59.

[30] Cho HS, Ha NC, Choi G, et al. Crystal structure of delta(5)-3ketosteroid isomerase from Pseudomonas testosteroni in complex with equilenin settles the correct hydrogen bonding scheme for transition state stabilization. J Biol Chem 1999; 274: 32863-8.

[31] Kim Y, Lim S, Kim Y. The role of a short strong hydrogen bond on the double proton transfer in the formamide-formic acid complex: theoretical studies in gas phase and in solution. J Phys Chem 1999; 103: 6632-7.

[32] Wu ZR, Ebrahimian S, Zawrotny ME, et al. Solution structure of 3oxo-delta5-steroid isomerase. Science 1997; 276: 415-8.

[33] Wohlfahrt G. Analysis of $\mathrm{pH}$-dependent elements in proteins: geometry and properties of pairs of hydrogen-bonded carboxylic acid side-chains. Proteins 2005; 58: 396-406.

[34] Liong EC, Dou Y, Scott EE, Olson JS, Phillips GN Jr. Waterproofing the heme pocket. Role of proximal amino acid side chains in preventing hemin loss from myoglobin. J Biol Chem 2001; 276: 9093-100.

[35] Fain AV, Berezovskii IN, Chekhov VO, Ukrainskii DL, Esipova NG. Double and bifurcated hydrogen bonds in alpha-helices of globular proteins. Biofizika 2001; 46: 969-77.

[36] Preissner R, Egner U, Saenger W. Occurrence of bifurcated threecenter hydrogen bonds in proteins. FEBS Lett 1991; 288: 192-6.

[37] Sarkhel S, Desiraju GR. N-H ${ }^{\cdots} \mathrm{O}, \mathrm{O}-\mathrm{H}{ }^{*} \mathrm{O}$, and $\mathrm{C}-\mathrm{H}^{*} \mathrm{O}^{\mathrm{O}}$ hydrogen bonds in protein-ligand complexes: strong and weak interactions in molecular recognition. Proteins 2004; 54: 247-59.

[38] Ben-Naim A, Ting KL, Jernigan RL. Solvent effect on binding thermodynamics of biopolymers. Biopolymers 1990; 29: 901-19.

[39] Olkhova E, Padan E, Michel H. The influence of protonation states on the dynamics of the NhaA antiporter from Escherichia coli. Biophys J 2007; 92: 3784-91.

[40] Teyra J, Pisabarro MT. Characterization of interfacial solvent in protein complexes and contribution of wet spots to the interface description. Proteins 2007; 67: 1087-95.

[41] DeLano WL. Unraveling hot spots in binding interfaces: progress and challenges. Curr Opin Struct Biol 2002; 12: 14-20. 